\title{
Efeitos da Desregulamentação na Extensão e no Grau de Integração do Mercado Brasileiro de Café
}

\author{
Fernando Tadeu Pongelupe Nogueira ${ }^{1}$ \\ Danilo R. D. Aguiar ${ }^{2}$
}

\begin{abstract}
Resumo: O setor cafeeiro passou por um processo de desregulamentação no período 1989/1990. Um fator externo, o final do Acordo Internacional de Café, aliado a um fator interno, a extinção do Instituto Brasileiro do Café, provavelmente aumentaram a competição e a integração no mercado brasileiro de café. Este artigo tentou identificar a extensão e a integração do mercado usando dados dos principais estados produtores e consumidores de café. Os resultados mostraram que a extensão do mercado brasileiro de café envolve estados de todas as regiões do país e todos os tipos de café (arábica e robusta). Além disso, o grau de integração aumentou após a desregulamentação do mercado, especialmente no mercado de café arábica.
\end{abstract}

Palavras-chave: eficiência de mercado; integração de mercado; integração espacial; desregulamentação do mercado.

\section{The Effects of Deregulation on the Extension and Degree of Integration of Brazilian Coffee Market}

\begin{abstract}
The coffee sector went through a deregulation process in the period 1989/1990. An external factor, the breakdown of the International Coffee Agreement, summed to an internal factor, the shutdown of the Brazilian Coffee Institute, probably increased the competition and the integration in the Brazilian coffee market. This paper tried to identify both market extension and market integration using data from the main states of the countries in terms of production and


consumption of coffee. Results showed that the extension of the coffee market involves states of all regions and all types of coffee (Arabic and Robusta). Besides, the degree of integration increased after market deregulation, especially in the market of Arabic coffee.

Keywords: market efficiency; market integration, special integration, market deregulation.

\section{Introdução}

No final do século passado, dois fatos caracterizaram o que este artigo denomina "desregulamentação" do mercado brasileiro de café. O primeiro foi um fato internacional: a suspensão do Acordo Internacional do Café (AIC), em 1989. O segundo foi um fato doméstico, a extinção do Instituto Brasileiro do Café (IBC), em março de 1990. Dessa forma, o setor cafeeiro brasileiro se viu diante de fortes mudanças organizacionais, estando, cada vez mais, sob influência dos chamados mercados globais. Uma possível conseqüência desse processo seria um maior grau de integração dos vários mercados regionais de café, tanto entre si como em relação aos principais mercados internacionais.

Os mercados integrados carregam informações mais precisas de preços e, portanto, favorecem a especialização e as decisões de comercialização dos produtores, contribuindo para a movimentação eficiente de produtos (GOODWIN e SCHROEDER, 1991). Dessa forma, é importante examinar se dado mercado é integrado e mensurar o grau de integração existente.

Vários estudos têm sido feitos com o objetivo de medir a integração de mercados. Em especial, GONZÁLEZ-RIVERA e HELFAND (2000) desenvolveram um método que permite identificar a extensão e o grau de integração de um mercado, aplicando-o ao mercado brasileiro de arroz. A extensão do mercado refere-se a sua dimensão e a suas fronteiras geográficas. O grau de integração do mercado é definido como o tempo de reação para que a relação de longo prazo consiga absorver um choque em todo o sistema, assim, a análise conjunta do impacto desses choques permite construir um ranking e ordenar os mercados com base nos tempos de reação.

O presente trabalho tem o objetivo geral determinar a extensão e o grau de integração nos mercados brasileiros de cafés arábica e robusta, antes e depois dos eventos que caracterizaram a desregulamentação do setor, utilizando como referência o método desenvolvido por GONZÁLEZ-RIVERA e HELFAND (2000). A hipótese que se propõe testar é a de que o grau de integração do mercado brasileiro de café aumentou após a desregulamentação. 


\section{Objeto de estudo}

Após ser introduzido no Brasil, no início do século XVIII, o café expandiuse pelo país, tendo chegado primeiro ao Rio de Janeiro, disseminando-se, em seguida, para Minas Gerais e São Paulo (OLIVEIRA, 1993). Atualmente, o Brasil produz os dois tipos de café comercializados no mundo, arábica e robusta, em mais de 10 estados da federação (Quadro1), com destaque para: Minas Gerais, São Paulo e Paraná, que praticamente só produzem café arábica; Espírito Santo e Bahia, que produzem entre 20 e 30\% de café arábica e o restante de robusta; e Rondônia e Mato Grosso, que só produzem café robusta (NOGUEIRA, 2005).

QUADRO 1 - PRINCIPAIS PRODUTORES DE CAFÉ NO BRASIL EM 2009 (EM TONELADAS E PERCENTAGEM)

\begin{tabular}{|l|r|r|}
\hline $\begin{array}{l}\text { Unidade da } \\
\text { Federação }\end{array}$ & Produção (t) & Parcela (\%) \\
\hline MG & 1.195 .488 & 49,14 \\
\hline ES & 619.655 & 25,47 \\
\hline SP & 193.920 & 7,97 \\
\hline BA & 176.851 & 7,27 \\
\hline RO & 93.819 & 3,86 \\
\hline PR & 89.213 & 3,67 \\
\hline GO & 18.802 & 0,77 \\
\hline RJ & 15893 & 0,65 \\
\hline MT & 7.653 & 0,31 \\
\hline CE & 3.289 & 0,14 \\
\hline Outros & 18.321 & 0,75 \\
\hline BRASIL & 2.432 .904 & 100,00 \\
\hline
\end{tabular}

FONTE: IBGE (2010).

Em termos mundiais, o Brasil é o maior produtor de café, seguido por Vietnã e Colômbia. O país já participou, no início do século XX, com quase $80 \%$ das exportações mundiais (NOGUEIRA, 2005), mas em 2009 as parcelas do Brasil foram de $46 \%$ no mercado de café arábica e $3 \%$ no mercado de café robusta (estimativas publicadas por CECAFÉ, 2010). Ainda em relação a 2009, os dados mostram que naquele ano o Brasil exportou o equivalente a 30.308.863 sacas de café, sendo 27.337.957 de café verde (1.114.676 de robusta e 26.223.281 de arábica) e 2.970.906 de café industrializado (106.809 de T\&M e 2.864.097 de café solúvel), sendo os principais destinos: Alemanha, 
EUA, Itália, Japão e Bélgica (dados do CECAFÉ, 2010). No caso específico do café verde, essas exportações se concentraram no porto de Santos (15,3\%) e Vitória (15,3\%). Com relação ao consumo, os principais consumidores são Estados Unidos, Europa, Japão, alguns países asiáticos e os próprios países produtores, dentre os quais, o Brasil se destaca como segundo maior consumidor mundial (NOGUEIRA, 2005).

A produção interna e o comércio internacional de café se desenvolveram sob forte aparato institucional. Segundo SCHIAVI (2003), internacionalmente, o mercado sofria forte regulamentação decorrente da existência dos Acordos Internacionais do Café (AIC), firmados no âmbito da Organização Internacional do Café (OIC) a partir de 1962. Tais acordos tinham como principal objetivo promover a sustentação de preços no mercado mundial, utilizando, principalmente, o sistema de quotas. Mas em 1989 houve o rompimento do AIC, com os países exportadores passando a tomar suas decisões livremente. Embora os países produtores ainda tenham reinstalado em 1993 o sistema de quotas, com a criação da Associação dos Países Produtores de Café (APPC), tal controle mostrou-se ineficaz e foi abolido em 2001. No âmbito doméstico, foi fundamental para o desenvolvimento do setor, até final da década de 1980, o Instituto Brasileiro do Café (IBC), órgão nacional que orientava a política para o setor e o tabelamento de preços no mercado nacional. Entretanto, essa forte regulamentação não estimulava a melhoria da qualidade do produto vendido no mercado doméstico, uma vez que o preço era fixo, não havendo remuneração maior para produto de melhor qualidade. Essas restrições foram eliminadas com a extinção do IBC em 1990.

Portanto, a partir da década de 1990, os principais mecanismos de regulação do setor foram extintos. Esse novo contexto permitiu à indústria maior autonomia para tomar decisões, acirrou a concorrência e permitiu que ganhos de competitividade fossem obtidos. Para tal, empresários dos vários segmentos da cadeia produtiva de café precisaram aprimorar seus mecanismos de obtenção e análise de informações visando tomar decisões mais eficientes, tanto sob o aspecto quantitativo quanto qualitativo. Informações sobre preços, oferta e demanda, área plantada, estoques, clima, entre outras, tornaram-se mais disponíveis, sendo que muitas dessas informações se concentraram em torno das operações de compra e venda de contratos futuros na Bolsa de Mercadorias e Futuros (BM\&F), em São Paulo, e na New York Board of Trade (NYBOT), em Nova Iorque. Neste contexto, os vários mercados regionais devem ter se tornado mais integrados uns aos outros, aumentando a eficiência da cadeia produtiva de café. 


\section{2. $O$ conceito de integração de mercado}

A origem da idéia de integração de mercado está na Lei do Preço Único (LPU), segundo a qual ao se abstraírem os custos de transferência, mercados regionais que são ligados por comércio e arbitragem terão um preço comum, único. Admitindo custos de transferências, a ação dos arbitradores assegura que os preços de bens homogêneos de quaisquer duas localidades difiram, no máximo, pelo custo de transferir o bem da região de menor preço para a região de maior preço. Quaisquer desvios deste são de natureza transitória.

Integração espacial de mercados diz respeito ao grau de co-movimentação dos preços em diferentes locais, medida pela correlação entre eles. Este é um conceito que independe da arbitragem, ou seja, independe de que os mercados estejam ligados diretamente por comércio. Por exemplo, os preços de uma commodity podem subir, em locais distintos, por razões que não dizem respeito diretamente à rede de negócios da commodity que une as diversas regiões. Assim, integração espacial de mercados é uma medida do grau pelo qual choques de demanda e oferta que surgem em uma região são transmitidos a outra.

Portanto, conforme salientam FLACKER e GOODWIN (2000), em mercados integrados poderia ocorrer transmissão de preços até mesmo de forma indireta. Suponha a existência de duas localidades "A" e "B", ambas fornecedoras de determinado bem para uma localidade "C". As localidades "A" e "B" poderiam estar fortemente integradas, apesar de não comerciarem diretamente. É justamente a ligação comercial com "C" que poderia fazer com que "A" e "B" estivessem integradas. Assim, choques de preços poderiam ser transmitidos, indiretamente, por meio da rede de comércio via ligações de comércio existentes entre regiões conectadas a esta.

\section{Dados e Procedimentos}

\subsection{Fonte de dados}

Foram utilizadas as informações mensais de preços de café recebidos pelo produtor, da FGV (Fundação Getúlio Vargas), das diversas unidades da federação, de janeiro de 1979 a fevereiro de 2004. Esses dados foram transformados em real, conforme procedimento indicado no site da FGV (http://fgvdados. fgv.br) e, em seguida, dolarizados, conforme cotações de câmbio do Banco Central do Brasil (http://bc.gov.br). Por fim, foram logaritmizados.

Dados de produção e população foram extraídos do Anuário Estatístico do 
Brasil, IBGE, mais precisamente dos anos de 1980, 1991 e 2000, para calcular o IAS - Índice de Auto-Suficiência. Dados de consumo foram obtidos das pesquisas de orçamentos familiares (POF) do IBGE.

\subsection{Métodos ${ }^{3}$}

\subsubsection{Testes preliminares}

Este estudo analisa a extensão do mercado e o grau de integração do mesmo. Para tal, alguns testes preliminares se fazem necessários, para se verificar se as séries apresentam as características requeridas. Inicialmente, são realizados testes de raiz unitária para se verificar se as séries são integradas de mesma ordem, o que é requerido para a realização dos testes de co-integração (VASCONCELOS e ALVES, 2000). Para tal, aplicou-se o Teste de Dickey-Fuller Aumentado (DFA), no qual se utilizam os valores tabulados por MACKINNON (1996). Estes testes são executados apenas para as séries incluídas na extensão preliminar do mercado, definida pelos Índices de Auto-Suficiência (IAS), conforme se descreve no próximo subitem.

A segunda etapa consiste em definir se as séries são co-integradas; caso sejam, identificam-se as relações de co-integração, que são as relações de equilíbrio no longo prazo. Utilizou-se o Teste de Johansen para co-integração, desenvolvido por JOHANSEN (1991 e 1995), o qual trabalha com vetores auto-regressivos (VAR).

\subsubsection{Determinação da extensão do mercado}

Seguindo o método de GONZÁLEZ-RIVERA e HELFAND (2000), o primeiro passo para determinar a extensão do mercado brasileiro de café é calcular o ÍNDICE DE AUTO-SUFICIÊNCIA (IAS). Para tal é necessário identificar o conjunto de localidades interligadas pelo comércio, o que pode ser feito por meio da estimativa dos fluxos de comércio para cada localidade. Como não estão disponíveis dados de comércio doméstico entre as diversas regiões do país, é necessário estimar esse fluxo de comércio. No caso específico, essa estimativa é feita em nível estadual, com base no nível de consumo anual de cada Unidade da Federação. Tal estimativa permite identificar as localidades que estão próximas da auto-suficiência, que são candidatas à descontinuação do comércio, bem como as que experimentaram inversão de comércio (exportadores que se tornaram importadores ou vice-versa). As localidades 3 Por limitação de espaço, optou-se por apresentar maiores detalhes apenas sobre os métodos utilizados para determinar a extensão e o grau de integração dos mercados. Os testes convencionais, tais como os testes de raiz unitária e de co-integração, não são apresentados detalhadamente. Para maiores detalhes, ver HARRIS (1995) ou ENGLE e GRANGER (1987). 
próximas à auto-suficiência e as que sofreram inversão de comércio serão excluídas do mercado a ser considerado. A justificativa para tal procedimento é que o modelo utilizado não contempla tais descontinuidades.

Na estimativa do consumo dos estados de café utilizaram-se informações sobre a população estadual e sobre o consumo per capita de café, sendo a multiplicação dessas informações utilizada como proxy do consumo estadual de café. Os cálculos forma feitos para os anos de 1987 e 1996, pois estes anos estavam em torno do marco divisório deste estudo (1990) e para os mesmos havia pesquisas de orçamentos familiares (POF) feitas pelo IBGE. Como as POFs não contemplavam regiões metropolitanas de todos os estados cobertos neste estudo, para os estados não cobertos pelas POFs foram consideradas as médias de consumo dos demais estados.

O IAS de cada estado foi calculado pela seguinte expressão:

$$
I A S_{f i, t}=\frac{\left(\operatorname{QTDPRODCAFE}_{f i, t}\right)}{\left(\operatorname{POPEST}_{f i, t} \cdot \mathrm{CONSPCCAFE}_{f i, t}\right)},
$$

em que IAS $\mathrm{Ifft}_{\mathrm{t}}$ ó índice de auto-suficiência de cada Unidade da Federação, no ano $t(t=1987$ ou $t=1996)$; QTDPRODCAFÉ ${ }_{u f, t}$, quantidade (em quilos) de café (em coco) produzida por cada Unidade da Federação, no ano t $(\mathrm{t}=1987$ ou $\mathrm{t}=1996)$; POPEST $_{\text {uf,t }}$, população estimada de cada Unidade da Federação, no ano $t(t=1987$ ou $t=1996)$; CONSPCCAFÉ ${ }_{\mathrm{uf}, \mathrm{t}}$, consumo (em quilos) per capita de café por Unidade de Federação, no ano $t(t=1987$ ou $t=1996)$.

Valores do IAS superiores a 1 (um) indicam que a Unidade da Federação é exportadora líquida da commodity de café. No caso de o IAS ser inferior a 1, tais valores indicam que a Unidade da Federação é importadora líquida de café. Caso os valores do IAS estejam próximos de 1, a Unidade da Federação estará próxima da auto-suficiência. Os cálculos do IAS foram feitos para dois anos para se verificar se houve inversões de comércio.

Neste trabalho, pretende-se identificar as Unidades da Federação brasileiras produtores de café que são conectadas, diretamente ou indiretamente, pelos negócios unidirecionais contínuos. Para isso, calcular-se-á o fluxo de negócios anual de café de cada localidade (estados brasileiros produtores de café).

A etapa seguinte depende da conclusão da primeira. Assim, após a identificação do conjunto de localidades que estão ligadas pelos negócios, busca-se pesquisar as localidades que compartilham um fator de integração comum. 
Segundo GONZÁLEZ-RIVERA e HELFAND (2000), em uma análise formal das implicações de um único fator de integração considera-se um vetor " $\mathrm{n}$ $\mathrm{X} 1$ ” não-estacionário I(1) de log-preços $p_{t}=\left\{p_{1 t}, p_{2 t}, \ldots, p_{t}\right\}$, em que $p_{i t}$ é o log-preço de uma commodity (no caso, log-preço do café das diversas Unidades da Federação do Brasil), no tempo "t”, no mercado "i”. Suponha que $p_{t}$ possa ser decomposto em dois componentes, como segue:

$$
p_{t}=A_{n X s} \cdot f_{t}+\tilde{p}_{i t}
$$

em que $f_{t}$ é um vetor "s x 1 " de "s" ( $\mathrm{s}<\mathrm{n}$ ) fatores de raiz unitária comum e $\widetilde{D}_{t}$, um vetor "n x 1 ” de componentes estacionários. Todo elemento no vetor $p_{t}$ poderá ser explicado por uma combinação linear de um número menor de fatores comuns de I(1), $f_{j t}$ (comporente permanente) mais um I(o) ou componente transitório (por exemplo, $\left.p_{i t}=\sum_{i=1}\left(a_{i j} . f_{j t}\right)+\tilde{p}_{i t}\right)$. As variáveis $p_{i t}$ movem-se juntas porque compartilham, no longo prazo, a mesma tendência estocástica. A representação (2) é conhecida como a representação do fator comum, e sua existência será garantida se, e somente se, houver " $n$ - $s$ " vetores de co-integração entre os elementos do vetor $p_{t}$ (no caso, log-preço do café das diversas Unidades da Federação do Brasil) - representação do Teorema de Granger, em ENGLE e GRANGER (1987).

O segundo resultado da representação do Teorema de Granger é que um sistema co-integrado pode ser escrito como um modelo de Vetor de Correção de Erro (VEC):

$$
\Delta P_{t}=\mathrm{m}+\Pi \cdot P_{t-1}+\Gamma_{1} \cdot\left(\Delta P_{t-1}\right)+\Gamma_{2} \cdot\left(\Delta P_{t-2}\right)+\ldots+\Gamma_{p-1} \cdot\left(\Delta P_{t-p+1}\right)+\mathrm{e}_{t},
$$

em que $\Delta$ é o operador de diferença; $\Delta P_{t}$, diferença do log-preço do café entre o período "t" e "t - 1"; $P_{t-1}$, log-preço do café no período " $\mathrm{t}$ - 1 " (defasado de um período); $\Delta P_{t-1}$, diferença do log-preço do café entre o período " $\mathrm{t}-1$ " e "t - 2 "; $\mathrm{e}_{t}$, erro aleatório (série ruído brando); e $\Gamma_{i}$ e $\Pi$, matrizes " $\mathrm{n} \mathrm{x}$ n" e $\Pi$ tem posto reduzido " $n$ - $s$ ".

A matriz $\Pi$ pode ser escrita como $\Pi=a \cdot b^{T}$, em que a é uma matriz " $n x$ (n - s)" de coeficientes e b , uma matriz “n x (n - s)" de vetores de co-integração. Usando esta expressão como $\Pi$, tem-se $\Pi \cdot P_{t-1}=\mathrm{a} \cdot \mathrm{b}^{T} \cdot P_{t-1}=\mathrm{a} \cdot Z_{t-1} \cdot \mathrm{O}$ termo de correção de erro, também conhecido como desequilíbrio de curto prazo, é $Z_{t-1}=\mathrm{b}^{T} \cdot P_{t-1}$, e a é a matriz de coeficientes de ajustamento. Os elementos da matriz $\mathrm{b}$ cancelam a raiz unitária comum em $P_{t-1}$ e, no longo prazo, ligam 
os movimentos dos elementos $P_{t}$.

No contexto deste trabalho, a definição de extensão de um mercado integrado requer que " $\mathrm{s}=1$ ", porque a procura é por localidades que compartilham a mesma informação de longo prazo. A representação do fator comum (2) torna-se $p_{i t}=a_{i 1} . f_{1 t}+\tilde{p}_{i t}, \quad i=1,2, \ldots, n$. Assim, segundo GONZÁLEZ-RIVERA e HELFAND (2000), "procurar por exatamente um fator comum é equivalente a procurar por ' $n-1$ ' vetores de co-integração". A procura por um conjunto mais amplo de localidades que compartilham " $n$ - 1 " vetores co-integrados é conduzida numa estrutura multivariada: o posto reduzido do VAR, proposto por JOHANSEN (1991). O teste de Johansen, para um número de vetores cointegrados, consiste no teste do posto de $\Pi$. O processo de testar pelo posto (rank) de П ocorre juntamente com a estimação dos vetores de co-integração e modelo de Vetor de Correção de Erros (VEC). Assim, contrariamente à metodologia de dois-estágios de Engle-Granger, a abordagem de Johansen é um procedimento de um estágio. Quando o número de relações de co-integração é identificado, têm-se estimado não só os vetores de co-integração, mas a dinâmica de curto prazo do sistema, dado pela equação (3).

GONZÁLEZ-RIVERA e HELFAND (2000) recomendaram, para determinar as localidades que pertencem ao mesmo mercado, iniciar com o máximo de localidades, "n", e testar, por "n-1", vetores de co-integração. Isto pode ser feito pelo teste da razão de verossimilhança, de Johansen (Johansen's likelihood ratio test), baseado na estatística traço. Se o número de vetores de co-integração for menor que " $n$ - 1" vetores, identificar-se-ão as localidades que deveriam ser removidas do sistema. Para isso, implementa-se um procedimento seqüencial que se inicia por um conjunto principal de "m" localidades $(\mathrm{m}<\mathrm{n})$ e testa-se um número de vetores de co-integração. Se o número for " $\mathrm{m}$ - 1", adiciona-se uma localidade ao conjunto. Com " $\mathrm{m}+1$ " localidades, ou a nova localidade compartilha uma tendência comum com as " $m$ " localidades anteriores, ou não. Caso compartilhe a mesma tendência comum, deverão ser encontrados " $m$ " vetores de co-integração. Se for descoberta uma tendência comum, esse procedimento deverá ser repetido, adicionando-se localidades, uma por vez; caso contrário, a localidade adicionada, que apresentava segunda tendência, deverá ser excluída, e o procedimento será repetido até o número de localidades se esgotar.

Após descobrir os “n - 1" vetores de co-integração, é necessário estimar o fator comum, que é feito pela metodologia proposta por GONZALO e GRANGER (1995), para estimar $f_{1 t}$. A metodologia de GONZALO e GRANGER (1995) é interessante porque o fator comum é associado a variáveis observáveis e permite a identificação das localidades que contribuem para o comportamento de longo prazo do preço de mercado. A estimação do fator comum é facilmente derivada da especificação do modelo (3). Duas condições são necessárias para identificar o fator comum. A primeira impõe que $f_{1 t}$ seja combinação 
linear dos elementos dos vetores de preços $\left\{p_{1 t}, p_{2 t}, \ldots, p_{t}\right\}$, então, $f_{1 t}$ é observável. A segunda impõe que, na equação (2), o componente transitório $\tilde{p}_{t}$ não-Granger cause o componente permanente $A . f_{1 t}$, no longo prazo. Assim, qualquer componente transitório não será transmitido para a previsão de longo prazo de $P_{t}$. Essa condição implica que, no modelo de Vetor de Correção de Erros (VEC), a única combinação linear de $\left\{p_{1 t}, p_{2 t}, \ldots, p_{t}\right\}$, tal que $\tilde{p}_{t}$ não tenha qualquer efeito no longo prazo de $P_{t}$, seja:

$f_{1 t}=\mathrm{a}_{\perp}^{T} \cdot P_{t}$,

(4)

em que $\mathrm{a}_{\perp}^{T} \mathrm{a}=0$. Essa condição de ortogonalidade significa que $\mathrm{a}_{\perp}^{T}$ elimina o termo de correção de erro $Z_{t-1}=\mathrm{b}^{T} \cdot P_{t-1}$ do modelo de Vetor de Correção de Erros (VEC), garantindo que não haja nenhum efeito do componente transitório sobre a previsão de longo prazo $P_{t}$. A equação (4) pode ser usada para identificar as localidades que contribuem para revelar a transmissão de informação de longo prazo.

\subsubsection{Determinação do grau de integração dos merca- \\ dos}

A medida que define o grau de integração pode ser obtida por meio das estimativas da equação (3), as quais seriam sumarizadas numa medida única. As funções de resposta de impulso têm sido usadas para medir o grau de integração, pois elas traçam o impacto, através do tempo, de um choque na localidade "j" no preço da localidade "i". Tais funções, no entanto, não são únicas quando os choques são correlacionados. Portanto, não é razoável esperar ter choques ortogonais, pois a série temporal de todos os preços é altamente correlacionada. A solução tem sido ortogonalizar os choques com a decomposição de Cholesky da matriz de co-variância de erros. Como esta decomposição varia com a ordem das variáveis do sistema, para cada ordem obtém-se diferente função de resposta de impulso. Assim, GONZÁLEZ-RIVERA e HELFAND (2000) propuseram uma alternativa que não impusesse uma ordenação no sistema.

O equilíbrio de longo prazo entre preços pode ser escrito por: 


$$
p_{1 t}=-\left(\frac{c_{i}}{\mathrm{~b}_{1 i}}\right)-\left(\frac{\mathrm{b}_{2 i}}{\mathrm{~b}_{1 i}}\right) \cdot p_{2 t}-\ldots-\left(\frac{\mathrm{b}_{\dot{i}}}{\mathrm{~b}_{1 i}}\right) \cdot p_{\hbar}+z_{i} ; i=1,2, \ldots, n-s
$$

em que $c_{i}$ é uma constante e todas as outras variáveis são definidas acima. Suponha que haja um choque em VAR que perturbe o equilíbrio de longo prazo, juntamente com $p_{t-1}$, que é $\left|Z_{t-1}\right| \neq 0$. Por (5) ser uma relação de cointegração, o vetor $z_{t}$ é estacionário. Isto implica que o efeito do choque será transitório e, eventualmente, irá acabar, enquanto o equilíbrio de longo prazo será estabelecido. Assim, GONZÁLEZ-RIVERA e HELFAND (2000) definiram o grau de integração como o tempo de reação para que cada uma das relações de equilíbrio de longo prazo absorvesse um choque em todo o sistema, confiando nos coeficientes estimados de a , b e $\Gamma$. Ao analisar o impacto desses coeficientes, torna-se possível construir um ranking consistente de mercados baseados no tempo de reação. GONZÁLEZ-RIVERA e HELFAND (2000) adotaram a metodologia de PESARAN e SHIN (1996), ao elaborarem perfis de persistência. Este trabalho também fará o mesmo, só que utilizará dados de preços de café dos diversos estados do Brasil, obtidos da FGV.

PESARAN e SHIN (1996) definiram o perfil de persistência como:

$$
H_{z}(k)=\operatorname{var}\left(Z_{t+k} / y_{t-1}\right)-\operatorname{var}\left(Z_{t+k-1} / y_{t-1}\right), k=0,1,2, \ldots
$$

em que $y_{t-1}$ é o conjunto de informações até o tempo "t-1"; $\operatorname{var}\left(Z_{t+k} / \mathrm{y}_{t-1}\right)$ , variância de $Z_{t+k}$, condicionada ao conjunto de informações; e "k", horizonte de tempo. A definição (6) é interessante para que var $\left(Z_{t+k} / y_{t-1}\right)$ seja a variância do passo à frente " $\mathrm{k}=1$ " do erro de previsão $Z_{t}$. Pode-se escrever $\operatorname{var}\left(Z_{t+k} / \mathbf{y}_{t-1}\right)=E\left\{\left[Z_{t+k}-E\left(Z_{t+k} / \mathbf{y}_{t-1}\right)\right] / \mathbf{y}_{t-1}\right\}^{2}$, em que $Z_{t+k}-E\left(Z_{t+k} / y_{t-1}\right)$ é o erro de previsão " $\mathrm{k}+1$ " de $Z_{t}^{t+k}$. De acordo com essa interpretação, a equação (6) indica que um perfil de persistência (persistence profile) é uma mudança na variância da previsão $Z_{t+k}$ com respeito à variância da previsão $Z_{t+k-1}$ com base no conjunto de informações $y_{t-1}$.

Da expressão (2) e considerando $z_{t}=c+\mathrm{b}^{T} \cdot P_{t}$, tem-se que $z_{t}=c+\mathrm{b}^{T} \cdot A \cdot f_{t}+\mathrm{b}^{T} \cdot \tilde{P}_{t}=c+\mathrm{b}^{T} . \tilde{P}_{t}$, a última igualdade segue de $\mathrm{b}^{T} \cdot A=0$, porque $z_{t}$ é estacionária. Conseqüentemente, tem-se que $H_{z}(k)=\mathrm{b}^{T}\left\{\operatorname{var}\left(\tilde{P}_{t+k} / \mathrm{y}_{t-1}\right)^{t}-\operatorname{var}\left(\tilde{P}_{t+k-1}^{\sim} / \mathrm{y}_{t-1}\right)\right\} . \mathrm{b}$, em que $\mathrm{k}=0,1,2, \ldots$ Para facilitar a comparação entre diferentes perfis, ordena-se $H_{z}(k)$. Para $\mathrm{k}=$ $\mathrm{O}, H_{3}(0)=\mathrm{b}^{T} \cdot\left\{\operatorname{var}\left(\tilde{P}_{t} / \mathrm{y}_{t-1}\right)-\operatorname{var}\left(\tilde{P}_{t-1} / \mathrm{y}_{t-1}\right)\right\} . \mathrm{b}=\mathrm{b}^{T} \cdot \Omega \cdot \mathrm{b}$. Seja a matriz diagonal G, que contem 0 inverso da raiz quadrada dos elementos da diagonal de $H_{z}(0)$ 
,$G=\operatorname{diag}\left\{\left[H_{1,1}(0)\right]^{1 / 2}, \ldots,\left[H_{n-s, n-s}(0)\right]^{1 / 2}\right\}$ O perfil de persistência orde-
nado é defirtido por

$$
H_{z}(k)=G \cdot H_{z}(k) \cdot G=\left\{h_{i, j}(k)\right\} ; k=0,1,2, \ldots
$$

sobre o impacto no tempo $\mathrm{k}=0$, o perfil $h_{i, i}(k)=1$ para $\mathrm{i}=1,2, \ldots, \mathrm{n}-\mathrm{s}$.

\section{Análise dos Resultados}

\section{1. Índice de auto-suficiência}

A Tabela 1 exibe os resultados calculados conforme a expressão (1). Considerando que algumas Unidades da Federação não produzem café ou não possuem dados levantados pelo IBGE (estas Unidades da Federação estão com valores zero no IAS, tanto de 1987 quanto de 1996, e NÃO na última coluna da Tabela 1) e que outras Unidades da Federação sofreram inversão de comércio (NÃO/INVER), estas foram descartadas para efeito da análise da extensão do mercado brasileiro de café, conforme explicado anteriormente. Assim, foram descartadas as seguintes Unidades da Federação: Roraima, Pará, Amapá, Tocantins, Rio Grande do Norte, Pernambuco, Sergipe, Rio Grande do Sul, Mato Grosso do Sul e Goiás. Portanto, a extensão do mercado brasileiro de café abrange, potencialmente, as seguintes Unidades da Federação: Rondônia, Acre, Amazonas, Maranhão, Piauí, Ceará, Paraíba, Alagoas, Bahia, Minas Gerais, Espírito Santo, Rio de Janeiro, São Paulo, Paraná, Santa Catarina, Mato Grosso e Distrito Federal. 
TABELA 1 - VALORES CALCULADOS DO IAS (ÍNDICE DE AUTO-SUFICIÊNCIA) PARA CAFÉ, POR UNIDADE DA FEDERAÇÃO, BRASIL, 1987 E 1996

\begin{tabular}{|c|c|c|c|}
\hline Estado & IAS 1987 & IAS1996 & SITUAÇÃO \\
\hline Rondônia & 39,1742 & 34,9646 & EXPORTADOR \\
\hline Acre & 0,8912 & 0,3065 & IMPORTADOR \\
\hline Amazonas & 0,0318 & 0,0420 & IMPORTADOR \\
\hline Roraima & 0,0000 & 0,0000 & $N \tilde{A} O$ \\
\hline Pará & 0,2322 & 2,3179 & NÃO/INVERSÃO \\
\hline Amapá & 0,0487 & 0,0000 & $N \tilde{A} O$ \\
\hline Tocantins & 0,0000 & 0,0004 & $N \tilde{A O}$ \\
\hline Maranhão & 0,0171 & 0,0005 & IMPORTADOR \\
\hline Piauí & 0,0079 & 0,0011 & IMPORTADOR \\
\hline Ceará & 0,8629 & 0,3173 & IMPORTADOR \\
\hline Rio Grande & 0,0013 & 0,0000 & $N \tilde{A} O$ \\
\hline $\begin{array}{l}\text { do Norte } \\
\text { Paraíba }\end{array}$ & 0,0148 & 0,0075 & IMPORTADOR \\
\hline Pernam- & 1,1100 & 0,2474 & NÃO/INVERSÃO \\
\hline $\begin{array}{l}\text { buco } \\
\text { Alagoas }\end{array}$ & 0,0149 & 0,0017 & IMPORTADOR \\
\hline Sergipe & 0,0000 & 0,0000 & $N \tilde{A} O$ \\
\hline Bahia & 7,6863 & 2,5366 & EXPORTADOR \\
\hline Minas & 19,2448 & 28,1812 & EXPORTADOR \\
\hline $\begin{array}{l}\text { Gerais } \\
\text { Espirito }\end{array}$ & 76,2111 & 92,0151 & EXPORTADOR \\
\hline $\begin{array}{l}\text { Santo } \\
\text { Rio de }\end{array}$ & 0,5550 & 0,3492 & IMPORTADOR \\
\hline $\begin{array}{l}\text { Janeiro } \\
\text { São Paulo }\end{array}$ & 6,6365 & 5,1491 & EXPORTADOR \\
\hline Paraná & 17,9391 & 4,5619 & EXPORTADOR \\
\hline Santa Cata- & 0,0462 & 0,0162 & IMPORTADOR \\
\hline rina ${ }_{\text {Rio Grande }}$ & 0,0000 & 0,0000 & $N \tilde{A O}$ \\
\hline $\begin{array}{l}\text { do Sul } \\
\text { Mato Gros- }\end{array}$ & 2,8083 & 0,4067 & NÃO/INVERSÃO \\
\hline $\begin{array}{l}\text { so do Sul } \\
\text { Mato Gros- }\end{array}$ & 10,9625 & 1,7224 & EXPORTADOR \\
\hline $\begin{array}{l}\text { SO Goiás } \\
\text { Go }\end{array}$ & 1,7909 & 0,4334 & 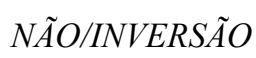 \\
\hline Distrito & 0,3901 & 0,3836 & IMPORTADOR \\
\hline Federal & & & \\
\hline
\end{tabular}

FONTE: Cálculos dos autores. 
Por não existirem séries de preços recebidos de café (dados da FGV) para Amazonas, Maranhão, Piauí, Paraíba, Alagoas, Santa Catarina e Distrito Federal, tais Unidades da Federação foram descartadas, restando como participantes do mercado brasileiro de café as Unidades da Federação: Rondônia, Acre, Ceará, Bahia, Minas Gerais, Espírito Santo, Rio de Janeiro, São Paulo, Paraná e Mato Grosso. Assim, o estudo prosseguiu com as séries de preços recebidos de café desses estados.

\subsection{Teste de raiz unitária}

Os testes de raiz unitária de Dickey-Fuller Aumentado (ADF), a partir de uma estimação com intercepto na equação de teste, utilizando defasagens de, no máximo, 24 períodos, tanto em nível quanto em primeiras diferenças (Tabelas A1 e A2 do apêndice), mostraram que as séries mensais logaritmizadas dos estados do Acre, Bahia, Ceará, Espírito Santo, Minas Gerais, Mato Grosso, Paraná, Rio de Janeiro, Rondônia e São Paulo são não-estacionárias em nível e estacionárias em primeira diferença, ou seja, são integradas de ordem 1, I(1). Portanto, os testes de co-integração foram feitos com as variáveis em primeiras diferenças.

\subsection{Teste de co-integração (teste de Johansen) e exten- são do mercado}

O teste de co-integração, de Johansen, é fundamental para evidenciar a extensão do mercado, pois, além de as séries terem de ser co-integradas, devem possuir apenas um fator comum, ou seja, devem ter " $n-1$ ” relações de co-integração.

No caso deste estudo, iniciou-se o procedimento operacional descrito na metodologia, ou seja, o teste de co-integração, com as séries dos estados de Minas Gerais e São Paulo. Optou-se por iniciar por estas séries por ser Minas Gerais o maior produtor de café do Brasil e por ser São Paulo o maior centro consumidor e e o local onde se encontra a bolsa de futuros em que são negociados contratos de café (BM\&F), além de se localizar neste estado o principal porto de exportação de café (Santos), o que gera um fluxo mais intenso de informações.

Na análise acrescentaram-se, um por vez, os estados remanescentes. A ordem de inclusão foi: Paraná, Espírito Santo, Rondônia, Mato Grosso, Bahia, Ceará, Rio de Janeiro e Acre. Os estados do Acre e Rio de Janeiro não foram consideradas como parte do mercado, visto que, ao incluir estes estados, necessitou-se de 20 a 23 defasagens ("lags") para conseguir “ $m$ - 1" vetores de co-integração, 
enquanto Minas Gerais, São Paulo, Paraná, Espírito Santo, Rondônia, Mato Grosso, Bahia e Ceará necessitaram de apenas uma defasagem ("lag") para conseguir " $m$ - 1" vetores de co-integração. Assim, os resultados para os estados participantes do mercado de café estão apresentados na Tabela 2.

TABELA 2 - TESTE DE JOHANSEN PARA AS SÉRIES MENSAIS LOGARITMIZADAS DE PREÇOS RECEBIDOS DE CAFÉ NOS ESTADOS DA FEDERAÇÃO RELACIONADOS - JANEIRO DE 1979 A FEVEREIRO DE 2004

\begin{tabular}{lll}
\hline \multicolumn{1}{c}{ Séries relacionadas $^{1}$} & $\begin{array}{c}\text { Defasagens nas } \\
\text { variáveis }\end{array}$ & \multicolumn{1}{c}{ Estatística do traço } \\
\hline & & Nenhuma: $302,55^{*}$ \\
& & eq. co-int.: $224,75^{*}$ \\
LNCE, LNBA, LNMT, & & eq. co-int.: $160,57^{*}$ \\
LNRO, LNES, LNPR, & 1 & 3 eq. co-int.: $113,07^{*}$ \\
LNSP, LNMG & 4 eq. co-int.: $73,57^{*}$ \\
& 5 eq. co-int.: $42,81^{*}$ \\
& 6 eq. co-int.: $22,05^{* *}$ \\
& 7 eq. co-int.: 5,80 \\
\hline
\end{tabular}

FONTE: Estimativas dos autores por meio do software Eviews.

Valores críticos:

1\%(*): 177,20 / 143,09 / 111,01 / 84,45 / 60,16 / 41,07 / 24,60 / 12,97.

5\%(**): 165.58 / 131,70 / 102,14 / 76,07 / 53,12 / 34,91 / 19,96 / 9,24.

As sete relações (vetores) de co-integração, conforme teste de co-integração (Tabela 2), normalizadas em relação a Minas Gerais, foram:

$$
\begin{aligned}
& \text { LNCE }=1,087632^{*} \text { LNMG - 0,353791; } \\
& \text { LNBA = 1,252350*LNMG - 0,619467; } \\
& \text { LNMT = 1,195890*LNMG - 0,754654; } \\
& \text { LNRO = 1,591475*LNMG - 2,190270; } \\
& \text { LNES = 1,354621*LNMG -1,565837; } \\
& \text { LNPR = 0,771084*LNMG +0,797561; } \\
& \text { LNSP = 1,204770*LNMG - 0,755952. }
\end{aligned}
$$

Com base na Tabela 2, nota-se que as unidades da federação integrantes do mercado são co-integradas e possuem sete relações de co-integração, que são as relações de equilíbrio de longo prazo, sendo seis estatisticamente significativas a $1 \%$ de significância e uma estatisticamente significativa a 5\% de significância. Tal fato sugere que os mercados dessas Unidades da Federação 
sejam eficientes na difusão de informações e operações de arbitragem. Assim, as informações disponíveis que afetam os preços no mercado brasileiro de café fluem entre os agentes dessa cadeia agroindustrial, disseminando-se nos demais mercados integrados.

Na seqüência, com o objetivo de confirmar a participação das séries no mercado, realizaram-se testes de Razão de Verossimilhança para as restrições relacionadas com os parâmetros ALFA e BETA, estimados por máxima verossimilhança por meio do procedimento de Johansen.

A Tabela 3 exibe os resultados dos testes de significância dos parâmetros BETA, enquanto a Tabela 4 apresenta os resultados dos testes de significância dos parâmetros ALFA. Na primeira linha da Tabela 3 tem-se o Ceará, cuja hipótese nula foi $B_{i 1}=0$, sendo $i=1,2, \ldots, 7$; na segunda linha, Bahia, cuja hipótese nula foi $\mathrm{B}_{\mathrm{i} 2}=0$, sendo $\mathrm{i}=1,2, \ldots, 7$; e assim sucessivamente. $\mathrm{Na}$ Tabela 4 a análise é similar, mas em relação aos parâmetros ALFA.

TABELA 3 - TESTE DE RAZÃO DE VEROSSIMILHANÇA PARA RESTRIÇÕES RELACIONADAS COM OS PARÂMETROS DE CO-INTEGRAÇÃO BETA, REFERENTE A CADA ESTADO PARTICIPANTE DO MERCADO - JANEIRO DE 1979 A FEVEREIRO DE 2004

\begin{tabular}{|c|c|c|}
\hline Hipótese nula $(\mathrm{BETA}=0)$ & Estatística-LR (razão de & Probabilidade \\
\hline 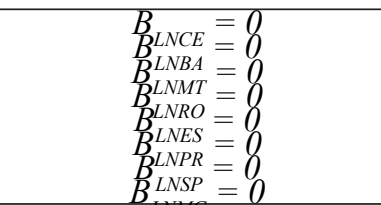 & $\begin{array}{l}45 \\
45,15 \\
41,57 \\
41,78 \\
45,05 \\
18,46 \\
43,63 \\
41,62\end{array}$ & $\begin{array}{l}0,000^{*} \\
0,000^{*} \\
0,000^{*} \\
0,000^{*} \\
0,000^{*} \\
0,011^{* *} \\
0,000^{*} \\
0,000^{*}\end{array}$ \\
\hline
\end{tabular}

FONTE: Estimativas dos autores por meio do software Eviews. 
TABELA 4 - TESTE DE RAZÃO DE VEROSSIMILHANÇA PARA RESTRIÇÕES RELACIONADAS COM OS PARÂMETROS DE CO-INTEGRAÇÃO ALFA, REFERENTE A CADA ESTADO PARTICIPANTE DO MERCADO - JANEIRO DE 1979 A FEVEREIRO DE 2004

\begin{tabular}{ccc}
\hline Hipótese nula (ALFA=0) & $\begin{array}{r}\text { Estatística-LR (razão de } \\
\text { máxima verossimilhança) }\end{array}$ & Probabilidade \\
\hline$A_{A N C E}=0$ & 51,86 & $0,000^{*}$ \\
$A_{L N B A}=0$ & 41,92 & $0,000^{*}$ \\
$A^{L N M T}=0$ & 42,94 & $0,000^{*}$ \\
$A_{L N R O}^{L N R}=0$ & 34,48 & $0,000^{*}$ \\
$A^{L N E S}=0$ & 16,64 & $0,019^{*}$ \\
$A_{L N P R}^{L N P R}=0$ & 20,32 & $0,005^{*}$ \\
$A_{L N H G}$ & 14,47 & $0,043^{*}$ \\
\hline
\end{tabular}

FONTE: Estimativas dos autores por meio do software Eviews.

Valores críticos: $\left(^{*}\right) 1 \%$ e $\left.{ }^{* *}\right) 5 \%$.

Os resultados da Tabela 3 mostram que todas as hipóteses nulas foram rejeitadas, a 1\% de significância, exceto para o Paraná, em que a hipótese nula foi rejeitada a $5 \%$ de significância.

Com relação ao teste de razão de verossimilhança para análise da significância dos coeficientes de ajustamento - ALFA -, verifica-se que todos os parâmetros ALFA, associados aos estados da federação, foram significativos a $1 \%$ de significância, exceto para Espírito Santo, São Paulo e Minas Gerais, cujas hipóteses nulas foram rejeitadas, a 5\% de significância.

Portanto, os resultados sugerem, também, que a extensão do mercado brasileiro de café abrange todos os tipos de café (arábica e robusta), pois participam do mercado regiões produtoras de café arábica (Minas Gerais, Paraná, São Paulo e Bahia) e de café robusta (Espírito Santo e Rondônia). Dos dez maiores produtores de café do Brasil, em 2009, ficaram fora do mercado apenas os estados de Goiás (descartado por não ser nem exportador, nem importador líquido) e Rio de Janeiro (que participou com menos de $1 \%$ da produção brasileira em 2009 e foi descartado porque a série de preço não se mostrou integrada, o que significa que este mercado é até certo ponto isolado dos demais). De qualquer forma, todos os estados que participaram com mais de $1 \%$ da produção nacional fazem parte de um único mercado.

\subsection{Grau de integração do mercado brasileiro de café}

A questão que se verifica aqui diz respeito à possível alteração do grau de 
integração no mercado brasileiro de café após a desregulamentação do setor cafeeiro. Para isso, calcularam-se os perfis de persistência antes e depois da desregulamentação, mais especificamente de janeiro de 1979 a fevereiro de 1990 e de março de 1990 a fevereiro de 2004, separando a série em dois períodos de pouco mais de 10 anos.

Nas Tabelas 5 e 6 estão listados os resultados do cálculo dos perfis de persistência antes da desregulamentação, tendo por base Minas Gerais e São Paulo, respectivamente. Nas Tabelas 7 e 8 estão os mesmos resultados após a desregulamentação do setor, para as mesmas bases.

TABELA 5 - ESTIMATIVA DOS PERFIS DE PERSISTÊNCIA - RESTRITO - EM RELAÇÃO A MINAS GERAIS - JANEIRO DE 1979 A FEVEREIRO DE 1990

\begin{tabular}{lc}
\hline Unidade da Federação & Perfil de persistência $(50 \%)$ \\
\hline CEARA & 0,6409 \\
BAHIA & 4,2925 \\
ESPIRITO SANTO & 5,2063 \\
RONDONIA & 6,4190 \\
SAOPAULO & 6,7247 \\
PARANA & 6,7730 \\
MATO GROSSO & 15,4336 \\
\hline
\end{tabular}

FONTE: Estimativas dos autores por meio do software Microfit.

TABELA 6 - ESTIMATIVA DOS PERFIS DE PERSISTENNCIA - RESTRITO - EM RELAÇÃO A SÃO PAULO - JANEIRO DE 1979 A FEVEREIRO DE 1990

\begin{tabular}{lc}
\hline Unidade da Federação & Perfil de persistência (50\%) \\
\hline CEARA & 0,6362 \\
BAHIA & 1,7796 \\
ESPIRITO SANTO & 1,8476 \\
RONDONIA & 5,5925 \\
PARANA & 5,7962 \\
MINAS GERAIS & 6,7247 \\
MATO GROSSO & 15,0838 \\
\hline
\end{tabular}

FONTE: Estimativas dos autores por meio do software Microfit.

TABELA 7 - ESTIMATIVA DOS PERFIS DE PERSISTÊNCIA - RESTRITO - EM RELAÇÃO A MINAS GERAIS - MARÇO DE 1990 A FEVEREIRO DE 2004

\begin{tabular}{lc}
\hline Unidade da Federação & Perfil de persistência (50\%) \\
\hline MATO GROSSO & 0,6850 \\
BAHLA & 0,9297 \\
SAOPAUL & 0,9627 \\
CEARÁ. & 1,4150 \\
PARANÁ & 1,4973 \\
ESPIRITOSANTO & 1,7129 \\
RONDONIA & 3,6242 \\
\hline
\end{tabular}


FONTE: Estimativas dos autores por meio do software Microfit.

TABELA 8 - ESTIMATIVA DOS PERFIS DE PERSISTÊNCIA - RESTRITO - EM RELAÇÃO A SÃO PAULO - MARÇO DE 1990 A FEVEREIRO DE 2004

\begin{tabular}{lc} 
Unidade da Federação & Perfil de persistência $(50 \%)$ \\
\hline MATO GROSSO & 0,7607 \\
BAHIA & 0,8653 \\
MUNAS GERAIS & 0,9627 \\
CEARÁ, & 1,6168 \\
PARANÁ & 1,8982 \\
ESPIRIT̃ SANTO & 2,1834 \\
RONDÕNIA & 3,3289 \\
\hline
\end{tabular}

FONTE: Estimativas dos autores por meio do software Microfit.

Conforme se pode observar nas Tabelas 5 a 8, os graus de integração calculados pelos perfis de persistência demonstram, tendo por base tanto Minas Gerais quanto São Paulo, que maior rapidez nos ajustamentos após a desregulamentação levou a maior grau de integração. É interessante o caso do Espírito Santo e de Rondônia, que produzem café robusta e ficaram mais integrados antes da desregulamentação do setor cafeeiro, ficando à frente, inclusive, de São Paulo (ou Minas Gerais - dependendo da base), Paraná e Mato Grosso, mas passaram para o último lugar após a desregulamentação, apesar de terem melhorado a velocidade dos ajustamentos. Assim, após a desregulamentação do mercado, Bahia, São Paulo (ou Minas Gerais, dependendo da base) e Paraná, principais produtores de café arábica, tornaram-se os estados mais integrados, o que sugere que o mercado de café arábica foi mais afetado do que o mercado de café robusta pelas mudanças organizacionais ocorridas no setor.

\section{Conclusões}

A análise permite concluir que o mercado brasileiro de café envolve os seguintes estados: Bahia, Ceará, Espírito Santo, Minas Gerais, Mato Grosso, Paraná, Rondônia e São Paulo. Ou seja, estados de todas as regiões brasileiras mostram-se integrados no mercado brasileiro de café. Ficou fora o estado do Rio de Janeiro, descartado porque sua série de preços não se mostrou integrada, o que indica ser este mercado isolado em relação aos demais. De qualquer forma, todos os estados que participam com mais de 1\% da produção nacional fazem parte de um único mercado.

Outro resultado relevante é que apesar de o Brasil produzir e comercializar cafés de tipos diferentes - café arábica e robusta -, a extensão do mercado brasileiro abrange regiões produtoras de ambos os tipos, ou seja, o mercado 
continua integrado, apesar de os cafés serem de tipos diferentes. Embora os diferentes tipos de café tenham preços diferentes, esses preços se movimentam de forma similar diante de choques de oferta ou demanda.

Por fim, os resultados dão suporte à hipótese de que a integração dos mercados aumentou ainda mais após a desregulamentação do setor de café, ressaltando os benefícios que a desregulamentação. Além da desregulamentação, e até certo ponto por ela influenciada, um forte processo de desenvolvimento e difusão na área de tecnologia de informações vem certamente propiciando maior integração nos mercados, conforme se evidenciou no item 2 deste artigo. Como conseqüência, maior tem sido o grau de eficiência do mercado brasileiro de café.

\section{Referências}

CECAFÉ. Balanço das exportações brasileiras de café. Conselho dos Exportadores de Café. Disponível em: http:// www.cecafe.com.br. (acesso em 20/o9/2010)

ENGLE, R.F., GRANGER, C.W.J. Co-integration and error-correction: representation, estimation and testing. Econométrica, v. 55, p. 251-276, 1987.

EVIEWS. User's guide. Irvine: QMS, 1997.656 p. (Versão 3.0).

FACKLER, P.L., GOODWIN, B.K. Spatial price analysis: forthcoming, handbook of agricultural economics. North-Holland, 2000. p. 1-59.

GONZÁLEZ-RIVERA, G., HELFAND, S.M. The extent, pattern and degree of market integration: a multivariate approach for the Brazilian rice market. American Journal of Agricultural Economics, September 2000.

GONZALO, J., GRANGER, C.W.J. Estimation of common long-memory components in the cointegrated systems. Journal of Business and Economic Statistics, v. 13 , n. 1, p. 27-35, 1995.

GOODWIN, B.K., SCHROEDER, T.C. Cointegration tests and spacial price linkages in regional cattle markets. American Journal of Agricultural Economics, p. 452-464, May 1991.

HARRIS, R.I.D. Using cointegration analisys in econometric modeling. new York: Prentice Hall, 1995. 176 p.

IBGE. Levantamento sistemático da produção agrícola - pesquisa mensal de previsão e acompanhamento das safras no ano civil. Rio de Janeiro, Instituto Brasileiro de Geografia e Estatística. Disponível em: ftp://ftp.ibge.gov.br/producao_agricola/ (acesso em 20/09/2010).

JOHANSEN, S. Estimation and hypothesis testing of co-integration vectores in gaussian vector autoregressive models. Econométrica, v. 59, p. 1551-1580, 1991.

JOHANSEN, S. Likelihood-based inference in co-integrated vector autoregressive models. Oxford: Oxford University, 1995. 
NOGUEIRA, F. T. P.; AGUIAR, D. R. D. Efeitos da desregulamentação na extensão e no grau ...

MACKINNON, J.G. Numerical distribution functions for unit root and cointegration tests. Journal of Applied Econometrics, v. 11, p. 601-618, 1996.

NOGUEIRA, F. T. P. Integração especial no mercado brasileiro de café. Viçosa, Universidade Federal de Viçosa, Tese de Doutorado em Economia Aplicada, 2005 .

OLIVEIRA, J. T. História do café no Brasil e no mundo. Belo Horizonte: Itatiaia, 1993. $440 \mathrm{p}$.

PESARAN, M.H., SHIN, Y. Cointegration and speed of convergence to equilibrium. Journal of Econometrics, v. 71, p. 117-143, 1996.

SAES, M. S. M.; JAYO, M. Competitividade do sistema agroindustrial do café. [dez. 2000]. ( www.cafe.com.br).

SCHIAVI, S. M. A. Café - Relatório Setorial Preliminar. FINEP, 2003. Disponível em http: // www.finep.gov.br/portaldpp/relatorio setorial/impressao relatorio. asp?lst setor $=15$ (acesso em 25/09/2010).

VASCONCELOS, M.A.S., ALVES, D. Manual de econometria. São Paulo: Atlas, 2000. $308 \mathrm{p}$. 
TABELA 1A -TESTE DE DICKEY-FULLER E DICKEY-FULLER AUMENTADO PARA AS SÉRIES MENSAIS LOGARITMIZADAS DE PREÇOS RECEBIDOS DE CAFÉ POR UNIDADE DA FEDERAÇÃO, EM NÍVEL - JANEIRO DE 1979 A FEVEREIRO DE 2004

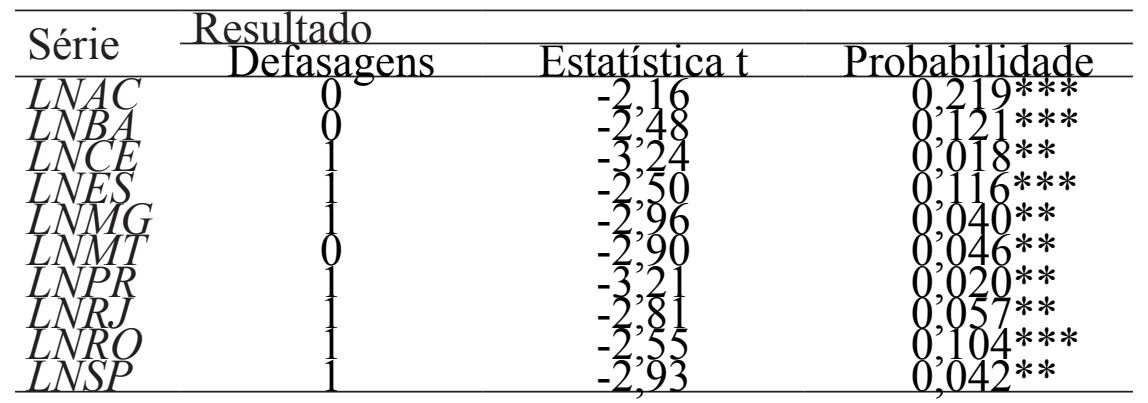

FONTE: Estimativas dos autores por meio do software Eviews.

Valores críticos (MacKinnon, 1996) para o e 1 defasagem ("lags"): $1 \%(*):-3,45 /-3,45 \mid 5 \%(* *):-2,87 /$ $-2,87 \mid 10 \%\left({ }^{* * *}\right):-2,57 /-2,57$.

TABELA 2A - TESTE DE DICKEY-FULLER E DICKEY-FULLER AUMENTADO PARA AS SÉRIES MENSAIS LOGARITMIZADAS DE PREÇOS RECEBIDOS DE CAFÉ POR UNIDADE DA FEDERAÇÃO, EM PRIMEIRA DIFERENÇA - JANEIRO DE 1979 A FEVEREIRO DE 2004

\begin{tabular}{lccc}
\hline Série & Resultado & & \\
\cline { 2 - 4 } Defasagens & Estatística t & Probabilidade \\
\hline$L N A C$ & 0 & $-18,47$ & $0,000^{*}$ \\
LNBA & 0 & $-16,15$ & $0,000^{*}$ \\
LNCE & 0 & $-14,60$ & $0,000^{*}$ \\
LES & 0 & $-12,74$ & $0,00^{*}$ \\
LNG & 0 & $-14,08$ & $0,000^{*}$ \\
$L N M T$ & 0 & $-16,87$ & $0,000^{*}$ \\
$L N P R$ & 0 & $-11,95$ & $0,000^{*}$ \\
$L N R J$ & 0 & $-14,01$ & $0,000^{*}$ \\
LNS & 0 & $-12,13$ & $0,000^{*}$ \\
LNSP & 0 & $-12,84$ & $0,000^{*}$ \\
\hline
\end{tabular}

FONTE: Estimativas dos autores por meio do software Eviews.

Valores críticos (MacKinnon, 1996) para o defasagem ("lags"): $1 \%\left(^{*}\right):-3,45\left|5 \%\left({ }^{* *}\right):-2,87\right| 10 \%\left({ }^{* *}\right)$ : $-2,57$. 
NOGUEIRA, F. T. P.; AGUIAR, D. R. D. Efeitos da desregulamentação na extensão e no grau ...

\section{(Footnotes)}

1 Modelo de Equação de Co-integração e Especificação do VAR: sem tendência determinística dos dados, com intercepto e sem tendência na equação de co-integração e sem intercepto no VAR. 